\title{
Prática de exercício físico ou esporte dos idosos jovens e longevos e o conhecimento dos mesmos em programas públicos: Pesquisa Nacional de Saúde 2013
}

\section{Physical exercise or sport of the old-adult and oldest old and the knowledge of them government programs: National Health Survey 2013}

\author{
Clarissa Biehl-Printes', Fabiane de Oliveira Brauner², Josemara de Paula Rocha ${ }^{3}$, Gabriela Oliveira ${ }^{4}$, \\ Jéssika Neris ${ }^{5}$, Bruna Rauber ${ }^{6}$, Ângelo José Bós ${ }^{7}$ \\ PhD. Institute of Geriatrics and Gerontology, Pontifical Catholic University of Rio Grande do Sul, Porto Alegre (RS), Brasil. <clarissaprintes@hotmail.com> \\ 2 Institute of Geriatrics and Gerontology, Pontifical Catholic University of Rio Grande do Sul, Porto Alegre (RS), Brasil. <braunerpro@gmail.com> \\ ${ }^{3}$ Institute of Geriatrics and Gerontology, Pontifical Catholic University of Rio Grande do Sul, Porto Alegre (RS), Brasil. <josemara.rocha@hotmail.com> \\ ${ }^{4}$ Institute of Geriatrics and Gerontology, Pontifical Catholic University of Rio Grande do Sul, Porto Alegre (RS), Brasil. <oliveira gabriela@hotmail.com> \\ ${ }^{5}$ Institute of Geriatrics and Gerontology, Pontifical Catholic University of Rio Grande do Sul, Porto Alegre (RS), Brasil. <neris-jehg3@hotmail.com> \\ ${ }^{6}$ Institute of Geriatrics and Gerontology, Pontifical Catholic University of Rio Grande do Sul, Porto Alegre (RS), Brasil. < brurauber@gmail.com> \\ MD PhD. Institute of Geriatrics and Gerontology, Pontifical Catholic University of Rio Grande do Sul, Porto Alegre (RS), Brasil.<angelo.bos@pucrs.br>
}

\section{ARTICLE INFO}

\section{Article history}

Received: 15/09/2016

Accepted: 09/12/2016

\section{Correspondent Author}

Clarissa Biehl Printes

Instituto de Geriatria e Gerontologia/PUCRS

Av. Ipiranga, 6681 pr. 81 sala 703.05

90619-900 Porto Alegre, RS, Brasil

<clarissaprintes@hotmail.com>

(C) 2016 All rights reserved

Editors

Alfredo Cataldo Neto

Paula Engroff

\begin{abstract}
RESUMO
Objetivo: descrever e relacionar a prática, participação, conhecimento e motivos de não-participação em relação às modalidades oferecidas pelos programas públicos de exercício físico ou esporte aos idosos jovens e longevos. Métodos: análise secundária de resultados da Pesquisa Nacional de Saúde 2013. Foram analisadas as frequências das respostas às questões P34 (prática de exercício físico ou esporte), P47 (conhecimento de algum programa público de exercício físico ou esporte), P48 (participação nesses programas), P49 (motivo de não participar desses programas) e P36 (exercício físico ou esporte que pratica com mais frequência) entre duas faixas etárias: idosos jovens (de 60 a 79 anos) e longevos (80 anos ou mais). O teste do qui-quadrado foi usado para verificar a distribuição das frequências considerando $p<0,05$. Resultados: $O$ estudo teve 9,679 idosos jovens e 1,498 idosos longevos. 78,9\% dos idosos brasileiros não praticam exercício físico com diferenças significativas entre idosos jovens $(77,3 \%)$ e longevos (89,3\%). Além disso $81,6 \%$ não conhecem a existência de programas e dos que conhecem a grande maioria não participa, $82,6 \%$. O motivo mais frequente de não-participação foi a falta de interesse (39\%) e a caminhada é a modalidade mais atrativa 58,4\%. Conclusões: $\bigcirc$ estudo revela uma baixa adesão dos idosos à prática de exercício físico ou esporte. Os indicadores mostram uma necessidade de ampliar a divulgação e diversificar os programas públicos de estímulo à prática do exercício físico ou esporte. São encorajados novos estudos sobre os mecanismos de comunicação utilizados na rede pública para divulgação desses programas.
\end{abstract}

PALAVRAS-CHAVE: Idoso; Idoso longevo; Exercício físico ou esporte; Programas públicos; Saúde Pública.

\begin{abstract}
Objective: describe and relate the practice, participation, knowledge and reason of non-participation in programs of exercise or sport offered by government programs to old-adult and the oldest old. Methods: secondary analysis of results of the Brazilian 2013 National Health Survey. We analyzed the frequency of responses to the questions P34 (physical exercise or sport), P47 (knowledge of a public program of physical exercise or sport), P48 (participation in these programs), P49 (reason not to participate in these programs) and P36 (physical exercise or sport that practices more often) in two age groups: old-adult ( 60 to 79 years) and oldest old ( 80 or more). The chi-square statistical test was used to verify the distribution considering $p<0.05$. Results: The study had 9,679 young elderly and 1,498 oldest old. Most Brazilians older-adult 78,9\% did not exercise with significant differences between old-adult (77,3\%) and oldest old $(89,3 \%)$. Furthermore, $81,6 \%$ did not know the existence of public programs and those who know the vast majority did not participate, $82,6 \%$. The most common reason for non-participation was the lack of interest (39\%) and walking is the modality more attractive $58,4 \%$. Conclusions: The study reveals a low compliance of the older-adult in physical exercise or sport practices. This study shows a necessity to publicize and increase the diversity of public programs to encourage the exercise or sport in these people. New studies into the communication mechanisms used in the public network for disclosure of these programs are encouraged.

KEYWORDS: Old-adult, Oldest old, Physical exercise and sport, Public programs, Public health.
\end{abstract}




\section{INTRODUÇÃO}

A inatividade física tem sido considerada um fardo para a saúde pública por representar um fator de risco condutor à instalação de uma variedade de doenças crônicas que se relacionam com a idade como: diabetes tipo 2, doenças cardiovasculares, osteodegenerativas, depressão, etc. ${ }^{1,2,3}$

Estudos anteriores descreveram que no Brasil, em 2008, mais de 13\% das mortes ocorridas foram atribuídas à inativididade física sendo então considerada a necessidade de incorporar a mensuração do exercício físico ou esporte em seu sistema de monitoramento e vigilância. ${ }^{4,5}$ Diante dos diferentes contextos em que o exercício físico ou esporte poderão ser realizados os programas municipais de estímulo para o exercício físico ou esporte são alvo de análise visto a amplitude de respostas que poderão alcançar nos cuidados da atenção primária à saúde da pessoa idosa e nas despesas com à saúde pública. ${ }^{3,6,7}$ Indicadores denominados atividade física de lazer; prática de exercício físico; esporte, representam as taxas das pessoas que se exercitam de acordo com as recomendações mínimas de saúde pública, isto é, atingir pelo menos 150 minutos semanais de atividade física regular para uma vida saudável. ${ }^{5,8}$

No Brasil $46 \%$ da população adulta é considerada insuficientemente ativa ou seja não atinge as recomendações mínimas semanais de atividade física e/ou exercício físico considerando o lazer, o trabalho e o deslocamento. ${ }^{5}$ Comparativamente com os países Europeus no topo temos a Inglaterra com a população mais preguiçosa $63 \%$ e noutra perspectiva os USA com $41 \%$ a não cumprir com as recomendações para prática de exercício físico diário de 30 minutos. ${ }^{9}$

Considerando que a saúde do idoso está amplamente relacionada com hábitos de vida e nível de atividade física e/ou exercício físico, encontra-se descrito na literatura especializada que, em idades mais avançadas, (acima dos 80 anos) um nível de atividade física inferior a s693 MET-min/sem (equivalente metabólico da tarefa) e uma capacidade de deslocamento inferior a $\leq 367,6$ m são fatores preditores para o idoso longevo vir a institucionalizar-se. ${ }^{10}$ Isto é, neste enquadramento, a inatividade física é considerada uma ameaça à condição de dependência. Apesar dos estudos aumentarem em cascata as evidências da importância dos exercícios na melhora da saúde do idoso, ainda se faz necessário conhecer como esta população encara a prática de exercício físico ou esporte, bem como o nível de conhecimento dos programas públicos municipais. ${ }^{11,12}$

No Brasil, em que a população envelhecida representa aproximadamente $11 \%$, com projeção para ultrapassar os $22,7 \%$ em 2050 , tem se assistido uma evolução gradual de programas direcionados à promoção e prevenção da saúde da pessoa idosa. ${ }^{13}$ No entanto, foi descrito, por estudo prévio de revisão sistemática, que os programas de exercício físico para pessoa idosa predominam em local acadêmico (universidade, laboratório), exisitindo poucos achados de trabalhos com a comunidade. Esse mesmo estudo critica sobre a escassez de estudos científicos de programas de exercício físico ou esporte, que atendam às dimensões propostas pelo modelo RE-AIM, utilizado no Brasil, como uma proposta de avaliação, que analisa tanto a validade externa quanto a validade interna de programas de saúde. Ainda esses autores concluiram que os achados evidenciaram limitações reduzindo assim a validade externa dos estudos. Salientam uma lacuna na ciência brasileira, no que se refere aos estudos de disseminação e implementação de programas de exercício físico ou esporte para idosos, que deveriam contribuir para o desenvolvimento de práticas e políticas públicas a serem incentivadas no país. ${ }^{14}$

Para o autor Chodzko-Zajko o idoso poderá sair em vantagem deste processo natural se for social, intelectual e fisicamente ativo. ${ }^{15}$ Sendo assim, o exercício físico tem sido considerado como o melhor contributo para um envelhecimento saudável e que não existe outro segmento da população que mais se beneficie do que os idosos. ${ }^{15,16,17}$ Por outro lado, os programas de estímulo para exercício físico ou esporte propostos por organismos públicos, têm sido considerados como poderosos recursos nos cuidados de atenção primária á saúde da pessoa idosa, no que toca a redução das despesas públicas, melhora da saúde, anos vividos com saúde, qualidade de vida e prevenção de situações de dependência. . $^{3,18,19}$

O objetivo do presente estudo foi descrever a participação de idosos em práticas de exercício físico ou esporte, como também seu grau de conhecimento sobre a existência ou não de programas municipais. Por fim os motivos que os levam a não participar e as modalidades oferecidas.

\section{MÉTODOS}

Trata-se de uma análise de dados secundários oriundos da Pesquisa Nacional de Saúde (PNS). A PNS foi um estudo transversal, descritivo e analítico, conduzido com uma amostragem de base populacional no Brasil, no ano de 2013, pelo Instituto Brasileiro de Geografia e Estatística (IBGE), com a colaboração do Ministério da Saúde (IBGE, 2014).

Ao todo foram entrevistados 60.202 participantes (IBGE, 2015). Sendo incluídos na análise todos 
os participantes idosos da pesquisa $(11,177)$. As perguntas de interesse foram questões do módulo P (Estilos de Vida): P34 (prática de exercício físico ou esporte), P47 (conhecimento de algum programa público de prática de exercício físico ou esporte), P48 (participação nesses programas), P49 (motivo de não participar desses programas) e P36 (exercício físico ou esporte que pratica com mais frequência). Para questão P36 foi feito um agrupamento por modalidades afins. Para a modalidade Caminhada foi incluida a caminhada ao ar livre e caminhada em esteira; para Ginástica foi incluido ginástica em geral e aeróbica; para as Atividades Aquáticas foi incluida a natação e hidroginástica; para as Modalidades Esportivas foram agrupadas o futebol, tênis, voleibol e basquetebol; por fim para outras modalidades foram agrupadas como Outros.

Os idosos foram agrupados em duas faixas etárias, idosos jovens (de 60 à 79 anos) e longevos (80 anos ou mais). Realizou-se análise descritiva das variáveis sociodemográficas e analítica, adotando a prática de exercício físico ou esporte, o tipo de modalidade, o conhecimento de programas públicos e o motivo de não participação, como variáveis categóricas dependentes e as faixas etárias como variáveis categóricas independentes. Utilizou-se o programa estatístico Epi Info $^{\text {тм }} 7$ para realizar as análises. Realizou-se o teste estatístico qui-quadrado para testar a associação da prática de exercício físico ou esporte entre idosos jovens e longevos, quanto a sua prática, no conhecimento de programas públicos de incentivo à prática de exercício físico ou esporte, na participação e motivos de não participação nos programas oferecidos e tipo de modalidades. Considerou-se nível de significância menor que 0,05 .

\section{RESULTADOS}

A Tabela 1 mostra a distribuição dos participantes idosos quanto ao sexo, estado civil e escolaridade. Entre os 11177 idosos (86,60\%) eram idosos (jovens), sendo $(58,44 \%)$ do sexo feminino e $(41,56 \%)$ do sexo masculino. Os outros (13,40\%) eram idosos (longevos), sendo $(64,49 \%)$ do sexo feminino e $(35,51 \%)$ do sexo masculino. Quanto ao estado civil os idosos jovens na maioria eram casados $(48,1 \%)$ seguidos de divorciados $(10,4 \%)$ ou separados $(3,9 \%)$. Nos idosos longevos também predominava o estado civil casado $(26,0 \%)$ seguido por divorciados $(5,0 \%)$ ou separados $(1,3 \%)$. A maior parte dos viúvos eram os idosos longevos $(57,3 \%)$. Com relação à escolaridade $(23,4 \%)$ de analfabetos eram idosos jovens uma frequência menor que o encontrado nos idosos longevos $(34,7 \%)$. Por outro lado foi encontrado uma paridade no ensino fundamental incompleto, nos jovens (45\%) e nos longevos $(44,7 \%)$. $\mathrm{O}$ mesmo ocorre no ensino fundamental completo com $(8,1 \%)$ nos jovens e $(6,6 \%)$ nos longevos. As diferenças ocorrem no ensino médio com $(12,8 \%)$ para os jovens e $(7,8 \%)$ para os longevos e no ensino superior com $(10,0 \%)$ nos jovens e $(6,0 \%)$ nos longevos.

Tabela 1. Distribuição dos participantes quanto ao sexo e estado civil.

\begin{tabular}{lccc}
\hline & Idosos jovens & Idosos longevos & Total \\
Sexo & & & \\
Feminino & $5656(58,44 \%)$ & $966(64,49 \%)$ & $6622(59,25 \%)$ \\
Masculino & $4023(41,56 \%)$ & $532(35,51 \%)$ & $4555(40,75 \%)$ \\
Estado Civil & & & \\
Casado & $4658(48,12 \%)$ & $390(26,03 \%)$ & $5048(45,16 \%)$ \\
Divorciado & $1008(10,41 \%)$ & $76(5,07 \%)$ & $1084(9,70 \%)$ \\
Separado & $377(3,90 \%)$ & $20(1,34 \%)$ & $397(3,55 \%)$ \\
Solteiro & $1207(12,47 \%)$ & $153(10,21 \%)$ & $1360(12,17 \%)$ \\
Viúvo & $2429(25,10 \%)$ & $859(57,34 \%)$ & $3288(29,42 \%)$ \\
Escolaridade & & & $2791(24,97 \%)$ \\
Analfabeto & $2271(23,46 \%)$ & $520(34,71 \%)$ & $5077(45,42 \%)$ \\
Fundamental Incompleto & $4406(45,52 \%)$ & $671(44,79 \%)$ & $886(7,93 \%)$ \\
Fundamental Completo & $787(8,13 \%)$ & $99(6,61 \%)$ & $1358(12,15 \%)$ \\
Médio & $1240(12,81 \%)$ & $118(7,88 \%)$ & $1065(9,53 \%)$ \\
Superior & $975(10,07 \%)$ & $90(6,01 \%)$ & 11177 \\
Total & $9679(86,60 \%)$ & $1498(13,40 \%)$ & \\
\hline
\end{tabular}

Fonte: Perfil dos idosos do RS, 2013. 
A Tabela 2 mostra a frequência na prática de exercícios físico ou esporte nos dois grupos de idosos. Observou-se que 8821 (78,9\%) dos 11177 idosos não praticavam exercício. Os idosos jovens 2195 (22,6\%) praticaram mais exercício físico ou esporte que os longevos $161(10,7 \%)$. A relação entre faixa etária e prática de exercício físico ou esporte foi significativa $(\mathrm{p}<0,001)$.

Observa-se, na Tabela 3, que a grande maioria dos idosos $(81,6 \%)$ teve pouco conhecimento da existência de programas públicos de exercício físico ou esporte no município em que residiam. A frequência dos que

Tabela 2. Frequência da Prática de Exercício Físico ou Esporte nos últimos 3 meses dos idosos jovens e idosos longevos.

\begin{tabular}{lccc}
\hline & Idosos jovens & Idosos longevos & Total \\
Não & $7484(77,32 \%)$ & $1337(89,25 \%)$ & $8821(78,92 \%)$ \\
Sim & $2195(22,68 \%)$ & $161(10,75 \%)$ & $2356(21,08 \%)$ \\
Total & $9679(86,60 \%)$ & $1498(13,40 \%)$ & 11177 \\
\hline
\end{tabular}

Tabela 3. Frequências do conhecimento e da participação e não-participação nos programas públicos de exercício físico ou esporte no município por idosos jovens e longevos.

\begin{tabular}{lccc}
\hline & Idosos jovens & Idosos longevos & Total \\
Não & $7820(80,79 \%)$ & $1310(87,45 \%)$ & $9130(81,69 \%)$ \\
Sim & $1859(19,21 \%)$ & $188(12,55 \%)$ & $2047(18,31 \%)$ \\
Participa & $335(18,02 \%)$ & $21(11,17 \%)$ & $356(17,39 \%)$ \\
Não participa & $1524(81,98 \%)$ & $167(88,83 \%)$ & $1691(82,61 \%)$ \\
Total & $9679(86,60 \%)$ & $1498(13,40 \%)$ & 11177 \\
\hline
\end{tabular}

conheciam e participavam foi baixa $(17,3 \%)$ sendo os idosos mais jovens os mais participativos (18,0\%). A proporção dos idosos longevos $(88,8 \%)$ que conheciam e não participavam foi muito próxima a dos idosos jovens (81,9\%). A relação entre faixa etária e conhecimento dos programas públicos foi significativa $(\mathrm{p}<0,001)$.

$\mathrm{Na}$ Tabela 4, o motivo mais frequente de não participação foi a falta de interesse (39\%), seguido de falta de tempo $(19,3 \%)$, problemas de saúde $(16,9 \%)$ e não ser perto do domicílio (13,6\%). Entre os longevos, a frequência de não realizar por falta de interesse, problemas de saúde e referir ter sido impedido de participar foi maior que a dos idosos jovens. Falta de tempo, distância da residência e falta de segurança predominou nos idosos mais jovens. A relação entre faixa etária e motivos de não participação foi significativa $(\mathrm{p}<0,001)$.

A Tabela 5 mostra as modalidades de exercício físico ou esporte que os idosos jovens e idosos longevos praticavam. Entre os 2306 praticantes 2151 (93,2\%) eram idosos jovens e 155 (6,7\%) eram longevos. Das modalidades mais praticadas a Caminhada $(58,0 \%)$ foi a que mais se destacou. Sendo que a maioria dos idosos longevos caminhavam $(63,8 \%)$. Na sequência específica das modalidades a Ginástica (16,7\%) foi a segunda opção seguida das Atividades Aquáticas $(7,4 \%)$. Os idosos jovens foram os que mais optaram pelas Atividades Esportivas (2,5\%) e que mais praticavam exercício fisico ou esporte $(93,2 \%)$.

Tabela 4. Frequência do motivo que leva a não participação nos programas públicos de exercício físico ou esporte no município por idosos jovens e idosos longevos.

\begin{tabular}{lccc}
\hline & Idosos jovens & Idosos longevos & Total \\
Não tenho interesse nas atividades oferecidas & $583(38,25 \%)$ & $78(46,71 \%)$ & $661(39,09 \%)$ \\
Não tenho tempo & $310(20,34 \%)$ & $17(10,18 \%)$ & $327(19,34 \%)$ \\
Problemas de saúde & $241(15,81 \%)$ & $45(26,95 \%)$ & $286(16,91 \%)$ \\
Não é perto do meu domicílio & $218(14,3 \%)$ & $13(7,78 \%)$ & $231(13,66 \%)$ \\
Outro & $129(8,46 \%)$ & $10(5,99 \%)$ & $139(8,22 \%)$ \\
O espaço não é seguro & $23(1,51 \%)$ & $1(0,6 \%)$ & $24(1,42 \%)$ \\
Fui impedido de participar & $20(1,31 \%)$ & $3(1,8 \%)$ & $23(1,36 \%)$ \\
Total & $1524(90,12 \%)$ & $167(9,88 \%)$ & 1691 \\
\hline
\end{tabular}

Tabela 5. Indicadores da frequência participativa dos idosos jovens e idosos longevos nas modalidades esportivas.

\begin{tabular}{lccc}
\hline & Idosos jovens & Idosos longevos & Total \\
Caminhada & $1249(58,07 \%)$ & $99(63,87 \%)$ & $1348(58.45 \%)$ \\
Ginástica & $357(16,60 \%)$ & $29(18,71 \%)$ & $386(16,74 \%)$ \\
Atividades Aquáticas & $160(7,44 \%)$ & $11(7,1 \%)$ & $171(7,41 \%)$ \\
Musculação & $97(4,51 \%)$ & $1(0,65 \%)$ & $98(4,25 \%)$ \\
Bicicleta & $69(3,21 \%)$ & $4(2,58 \%)$ & $73(3,17 \%)$ \\
Atividades Esportivas & $54(2,52 \%)$ & $1(0,65 \%)$ & $55(2,38 \%)$ \\
Outro & $165(7,67 \%)$ & $10(6,46 \%)$ & $175(7,59 \%)$ \\
Total & $2151(93,27 \%)$ & $155(6,73 \%)$ & 2306 \\
\hline
\end{tabular}




\section{DISCUSSÃO}

Tendo em vista a expansão da população idosa no Brasil é destacada a importância dos programas públicos de estímulo ao exercício físico ou esporte para a pessoa idosa. O perfil demográfico do idoso brasileiro, obtido na PNS de 2013, permite constatar que a maioria desta população não pratica exercício físico ou esporte, isto é, não cumpre com os níveis de recomendação de prática de atividade física regular. ${ }^{5,20} \mathrm{Em}$ proporção são os idosos jovens os que mais se exercitam.

No contexto mundial essa transição demográfica demonstra que a proporção de idosos com 80 anos ou mais vem aumentando consideravelmente, trazendo implicações importantes, principalmente na área da saúde, devido à maior frequência de morbidades e maior incidência de declínio funcional. ${ }^{13,21} \mathrm{O}$ aumento da expectativa de vida traz consigo a preocupação com a saúde, pois as doenças ligadas ao processo de envelhecimento podem levar ao aumento dos custos assistenciais de saúde. ${ }^{22}$

Os programas públicos de estímulo à prática de exercício físico ou esporte são estratégias de ação muito eficazes no combate ao sedentarismo, controle das DCNT (doenças crônicas não transmissíveis), oportunidade de participação e inclusão social. De acordo com os dados da PNS, a grande maioria dos idosos brasileiros desconhece a existência destes tipos de programas. Parece existir uma tímida divulgação deles junto à comunidade já que se tem assistido um crescimento gradual deste tipo de intervenção. Como tem sido demonstrada, a implementação de programas públicos permite analisar a qualidade e viabilidade de execução, como recurso promotor e preventivo da saúde, permitindo o conhecimento de evidências no controle das despesas em saúde pública. ${ }^{3}$ Uma futura pesquisa detalhada sobre os mecanismos de comunicação utilizados na rede pública deveria explorar, com maior rigor, o nível de conhecimento dos programas públicos municipais, para então dar por confirmada esta hipótese. Assim, consideramos neste estudo uma limitação sobre a real existência de programas de exercício físico ou esporte, pois, se os mesmos existem, parece não serem eficientes na sua divulgação para atingir o nível de conhecimento dos idosos.

Um outro aspecto preocupante é a pouca frequência de participação pela totalidade dos idosos nos programas, menos de $20 \%$ dos que conhecem afirmaram aderir, porém uma proporção espantosamente elevada, verificada nas duas faixas etárias, não participa, apesar de conhecer. Além disso, os idosos jovens participam mais do que os longevos. Como foi descrito anteriormente, a localização e características internas das propostas de intervenção poderão influenciar na participação. ${ }^{14}$

Em parte, diferente do que foi exposto pelo estudo prévio de revisão sistemática, os dados da PNS revelaram que a falta de interesse dos idosos é a principal razão para não participar, sendo curioso já que tem sido descrito que a população idosa gosta de se exercitar, como caminhar e obter benefícios à saúde. ${ }^{14,23}$ No entanto, os dados mostram que, de fato, a caminhada é uma forte opção, sendo os longevos os que mais apreciam, seguida da ginástica, e atividades aquáticas. Essas modalidades recuperam a função física geral e repercutem diretamente nos estados de aerobiose, força, resistência, mobilidade, flexibilidade e equilíbrio que, por sua vez, influenciam na integridade funcional sentida nas atividades da vida diária. As atividades esportivas como futebol, tênis, voleibol e basquetebol tiveram muito baixa aderência em ambas as faixas etárias. Nesse aspecto chamamos a atenção para a prática dos jogos adaptados a serem ajustados aos idosos e sedentários. A adaptação do jogo para o idoso permite sua participação, independente de sua aptidão física ou habilidades motoras. ${ }^{24}$

Enquanto os idosos jovens atribuem a ausência nos programas também por falta de tempo, problemas de saúde e distância do domicílio, os longevos se enquadram mais com o que foi descrito no estudo nacional de revisão sobre programas de exercício físico ou esporte para o idoso de 2015, alegam limitaremse por problemas de saúde e referem terem sido impedidos de participar. ${ }^{14}$ Dos trabalhos encontrados nessa revisão, foram os idosos jovens que constituiram a quase totalidade das amostras, ainda que o estudo tenha sido extensivo a todas as faixas etárias acima dos 60 anos como critério de inclusão. De fato, estas evidências alertam para uma necessidade mais alargada às faixas etárias das propostas de estímulo à prática de exercício físico ou esporte. Há uma emergente necessidade de promover a educação para saúde do idoso e criar ambientes favoráveis, capazes de gerar oportunidade de participação e inclusão desta população sem diferenciação etária e demais características. Unicamente deverão estar adequados às características e necessidades destas pessoas. A título de exemplo, citamos a estratégia do programa Tomando Controle da Sua Saúde, promovido pela Organização Pan Americana de Saúde uma proposta que integra a educação e um conjunto multidisciplinar de práticas em que o indivíduo aprende a cuidar e ser responsável pela sua própria saúde. ${ }^{25}$ No contexto da pessoa idosa interessa que ela aprenda e compreenda que exercício físico ou esporte não correspondem unicamente a uma 
atividade esportiva ou de lazer a ser integrada na sua rotina e sim uma medida preventiva de manutenção da saúde com baixo custo.

Grande parte dos recursos de saúde, como visitas ao consultório, hospitalização e medicamentos são exigidos pelos idosos. ${ }^{3}$ Na prática clínica a recomendação médica para aumentar a atividade física e ou exercício físico tem sido um recurso promotor visto que aumentos moderados revelaram melhoras na qualidade de vida em idosos jovens. ${ }^{26,27}$

O único estudo encontrado que analisou a fundo o cumprimento dos critérios de qualidade e aplicabilidade dos programas de estímulo à práticas de exercício físico ou esporte no Brasil critica a totalidade das propostas na carência de análise da dimensão eficácia/ efetividade através do custo-efetividade tão útil em saúde pública. ${ }^{14} \mathrm{~A}$ eficácia/efetividade representa o resultado final da implementação de um programa, onde os efeitos positivos e negativos, a qualidade de vida e os resultados econômicos são verificados. ${ }^{14,28}$

Voltados a este contexto, descrevemos uma análise positiva realizada por poucos estudos. ${ }^{14,29,30}$ Concluem os mesmos sobre a importância da inclusão de programas de estímulo à prática de exercício físico ou esporte como estratégia econômica na poupança em despesas públicas, reforço do papel do exercício físico como recurso preventivo à saúde e otimização dos serviços de saúde na atenção primária de pessoas idosas e pacientes de DCNT por ações multidisciplinares entre o médico e educadores físicos. Em contrapartida, como foi descrito ao analisar, o custo-efetividade, em programas auto-supervisionados, ou seja em que as pessoas/ pacientes apenas seguiam o aconselhamento médico para incluírem a prática de exercício físico regular na rotina não foram economicamente tão efetivos como os supervisionados, isto é, acompanhados por educador físico num local propício. ${ }^{31}$

É evidente que a aplicação de programas públicos deve controlar também outras dimensões como: alcance (número absoluto, proporção do público-alvo atendido e sua representatividade no programa, e avaliação das características do público-alvo); adoção (proporção e perfil da equipe, dos praticantes e dos planos adotados na intervenção); implementação (é a fidelidade da intervenção por parte das organizações e dos sujeitos); e manutenção (quanto o programa se estendeu após sua implementação). 14,27,28

A educação, promoção e divulgação dos programas públicos de estímulo ao exercício físico ou esporte no Brasil devem ser encarados como estratégia nos cuidados de saúde primária. Isto está bastante evidente nestes resultados da PNS de 2013. Devem ser incentivados pelos orgãos públicos para que a sua ocorrência não fique tão demarcada no âmbito acadêmico. Uma prática organizacional na saúde municipal deve estar mais ativa e preparada para atender a comunidade idosa na esfera global da heterogeneidade das condições.

\section{CONCLUSÕES}

Concluimos que há uma baixa adesão dos idosos em práticas de exercício físico ou esporte ainda menos frequente entre os longevos. A falta de interesse foi a principal razão para não participarem e a caminhada a modalidade mais atrativa. Os indicadores revelam, ainda, uma necessidade de ampliar a divulgação dos programas públicos de estímulo à prática do exercício físico ou esporte para as pessoas idosas. A falta de diversidade também impõe condições e restrições para diferentes faixas etárias. Futuros estudos são encorajados a explorarem sobre os mecanismos de comunicação utilizados na rede pública para divulgação destes programas.

\section{REFERÊNCIAS}

1. Allender S, Foster C, Scarborough P, et al. The burden of physical activity-related ill health in the UK. Journal of epidemiology and community health. 2007;61:344-8.

2. Bassuk SS, Manson JE. Epidemiological evidence for the role of physical activity in reducing risk of type 2 diabetes and cardiovascular disease. J Appl Physiol. 2005;99: 1193-204.

3. Gusi N, Reyes MC, Gonzalez-Guerrero JL, et al. Cost-utility of a walking programme for moderately depressed, obese, or overweight elderly women in primary care: a randomised controlled trial. BMC Public Health. 2008;8:231.

4. Lee IM, Shiroma EJ, Lobelo F, et al. Effect of physical inactivity on major non-communicable diseases worldwide: an analysis of burden of disease and life expectancy. Lancet. 2012;380:219-29.

5. Mielke GI, Hallal PC, Rodrigues GBA, et al. Physical activity and television viewing among Brazilian adults: National Health Survey 2013. Epidemiol. Serv. Saúde. 2015;24: 277-86.

6. Caspersen C, Powell K, Christenson G. Physical activity, exercise, and physical fitness: definitions and distinctions for health-related research. Public Health Rep. 1985;100: 126-31.

7. American College of Sports Medicine, Chodzko-Zajko WJ, Proctor DN, et al. American College of Sports Medicine position stand. Exercise and physical activity for older adults. Med Sci Sports Exerc. 2009;41:1510-30.

8. Zaitune MPA. Comportamentos de saúde de idosos: resultados de inquérito populacional [Tese de Doutorado]. Campinas: Universidade Estadual de Campinas; 2010.

9. UK.England for commissioning the ukactive Research: Steps to solving inactivity 2004 [Internet]. England; 2014 [cited 2016 Aug 28]. Available from:<http:/www.ukactive.com/ downloads/managed/Final Final Final Steps to Report. $\mathrm{pdf}>$. 
10. Pereira CL, Fernandes J, Raimundo A, et al. Increased Physical Activity and Fitness above the 50th Percentile Avoid the Threat of Older Adults Becoming Institutionalized: A Cross-sectional Pilot Study. Rejuvenation Res. 2016;19: 13-20.

11. Faber MJ, Bosscher RJ, Chin AP, et al. Effects of exercise programs on falls and mobility in frail and prefrail older adults: a multicenter randomized controlled trial. Arch Phys Med Rehabil. 2006;87:885-96.

12. Chou CHH, Wang CL, Wu YT. Effect of exercise on physical function, daily living activities, and quality of life in the frail older adults: a meta-analysis. Arch Phys Med Rehabil. 2012;93:237-44.

13. Instituto Brasileiro de Geografia e Estatística, Diretoria de Pesquisas, Coordenação de População e Indicadores Sociais. Síntese de Indicadores Sociais: uma análise das condições de vida da população brasileira 2013: Estudos e Pesquisas; Informação Demográfica e Socioeconômica, n. 32. Rio de Janeiro (Brasil). IBGE; 2013.

14. Squarcini CFR, Rocha SV, Munaro HLR, et al. Physical activity programs for elderly persons: an evaluation of Brazilian scientific production using the RE-AIM framework. Review Article. Rev. Bras. Geriatr. Gerontol.2015;18: 909-20.

15. Chodzko-Zajko WJ. In: Jessie JC, Rose JDP. Physical Activity Instruction of Older Adults. Psychological and Sociocultural Aspectos of Physical Activity for Older Adults. US:Human Kinetics. 2005. Chapter 3.

16. Bortz W, Bortz S. Prevention, nutrition, and exercise in the aged. In: Carstensen LL, Edelstein BA, Dornbrand L. The pratical handbook of clinical gerontology. Dornbrand (Eds.). California: Sage Publications.1996.

17. Evans W. Exercise training guidelines for the elderly. Medicine and Science of Sports and Exercise. 1999;31:12-7.

18. Sevick MA, Dunn AL, Morrow MS, et al. Cost-effectiveness of lifestyle and structured exercise interventions in sedentary adults: results of project ACTIVE. Am J Prev Med. 2000; 19:1-8

19. Herdman M, Badia X, Berra S. EuroQol-5D: a simple alternative for measuring health-related quality of life in primary care. Aten Primaria. 2001;28:425-30.

20. Nelson ME, Rejeski WJ, Blair SN, et al. Physical and public health in older adults: recommendation from the American College of Sports Medicine and the American Heart Association. Med Sci Sports Exerc. 2007;39:1435-45.
21. Lourenço TM, Lenardt MH, Kletemberg FD. Capacidade Funcional no Longevo. Uma revisão integrativa. Rev. Gaúcha Enferm. 2012;33:176-85.

22. Papalia DE, Olds SW, Feldman RD. Desenvolvimento humano. 10 $0^{\mathrm{a}}$ ed. Porto Alegre: Artmed; 2009.

23. Gusi N, Prieto J, Forte D, et al. Needs, interests, and limitations for the promotion of health and exercise: a web site for sighted and blind elderly people. Educational Gerontology. 2008;34:1-13.

24. Brasil (RS). SEL: Secretaria Estadual do Esporte e do Lazer do Rio Grande do Sul. Regulamento oficial do XV Jogos de Integração do Idoso do RS, ano 2013 [Internet]. Porto Alegre; 2013 [acesso em 30 ago. 2016]. Disponível em: <http://www.sel.rs.gov.br/upload/1377868327 Regulamento\%20oficial\%20\%20XV\%20Jogos\%20de\%20 Integracao\%20do\%20Idoso\%20do\%20RS\%202013\%20 30-08-2013.pdf $>$.

25. Organización Panamericana de la Salud. Promoción de la salud: una antología. Washington, D.C.: OPS, 1996.

26. King AC, Rejeski WJ, Buchner DM. Physical activity interventions targeting older adults. A critical review and recommendations. American Journal of Preventive Medicine.1998;15:316-33.

27. Guallar-Castillon P, Santa-Olalla PP, Banegas JR, et al. Physical activity and quality of life in older adults in Spain. Medicina Clinica. 2004;123:606-10.

28. Glasgow RE, Vogt TM, Boles SM. Evaluating the public health impact of health promotion interventions: the RE-AIM framework. Am J Public Health. 1999;89: 1322-7.

29. Munro J, Brazier J, Davey R, et al. Physical activity for the over-65s: could it be a cost-effective exercise for the NHS? Journal of Public Health Medicine. 1997;19:397-402.

30. Stevens W, Hillsdon M, Thorogood M, et al. Cost-effectiveness of a primary care based physical activity intervention in 45 . 74 year old men and women: a randomised controlled trial. British Journal of Sports Medicine. 1998;32:236-41.

31. Tully MA, Cupples ME, Chan WS, et al. Brisk walking, fitness, and cardiovascular risk: a randomized controlled trial in primary care. Preventive Medicine 2005;41: $622-8$.

32. Almeida FA, Brito FA, Estabrooks PA. Modelo REAIM: tradução e adaptação cultural para o Brasil. REFACS 2013;1:6-16. 